\title{
An improvement of the Lagrangian analysis method based on particle velocity profiles
}

\author{
Yuanyuan Ding* \\ Faculty of Mechanical Engineering \& Mechanics, Ningbo University, Ningbo 315211, PR China
}

\begin{abstract}
In general, techniques used in studies on dynamic behaviour of materials could be classified into two categories, namely the split Hopkinson pressure bar technique (SHPB) and the wave propagation technique (WPT). Lagrangian analysis method is one of the most famous methods in WPT. The traditional Lagrangian analysis based on the particle velocity wave-profiles measurements should consider a boundary condition, because it involves integral operations. However, the boundary stress data in some cases cannot be detected or determined by the experimental measures. To tackle this situation, this paper presents a modified Lagrangian analysis method which does not involve the boundary stress computation. Starting from the path-lines method and utilizing the zero-initial condition, the material constitutive stress-strain curves under high strain-rates is deduced from only observing the particle velocity curve measurements. The dynamic stress/strain wave-profiles of the PMMA material, as a paradigm, are numerically studied using the proposed method, which are well in agreement with the theoretical result using the method of characteristics, which confirms the reliability and validity of the presented method.
\end{abstract}

\section{Introduction}

In the studies of dynamic response of materials under impact and blast loadings, two dynamic effects, i.e. the inertia effect and the strain rate effect, should be taken into consideration. However, those two effects are often inter-coupled. On the one hand, the analysis of stress wave propagation must be based on the premise of the dynamic constitutive of the materials, on the other hand, the propagation of stress waves in the material cannot be neglected when studying the dynamic constitutive of materials at high strain rates. At present, there are two methods to study the dynamic constitutive relationship of material under high strain rate. The first is the Split Hopkinson Pressure Bar (SHPB) technique, which can decouple the strain wave effect and the strain rate effect in the experiment, and has been widely used in many solid materials. The other is the wave propagation method, by which the constitutive relationship of materials can deduced combining the wave propagation information in the specimen [1-2].

Due to the long elastic bar and short specimen used in SHPB, the strain rate effects and the wave effects are cleverly uncoupled. However, the validity of SHPB results depends on two basic assumptions, namely the assumption of one-dimensional stress wave propagation and the assumption of stress uniformity along the specimen [3]. Those two basic assumptions are not always satisfactory. For example, porous materials can not satisfy the assumption of stress uniformity along the specimen in SHPB for their nonnegligible local deformation. Thus, the second method, wave propagation method, should be applied to investigate the dynamic stress-strain relation of materials.

Lagrangian analysis method, one of the wave propagation technique, was first proposed by Fowles, Cowperthwaite and Williams [4-5], and further developed by Grady [6]. Since the 1970s to the 1980s, a lot of research work [7-9] was carried out based on the Lagrangian analysis method for its no constitutive assumption in advance. In the Lagrangian analysis method, the dynamic constitutive relation of materials can be deduced directly from a series of physical quantity measurements regardless of the two coupled dynamic effects, because the interaction of the inertia effect and strain-rate effect is naturally and implicitly considered in the wave propagation technique. Li [7] revealed the rate-dependent constitutive relation characteristic of PMMA by Lagrangian analysis. Chai et al. [8] systematically discussed the error development in Lagrangian analysis method, and proposed advices to reduce the error. Wang et al. [9] proposed a new method combining the Lagrangian analysis with the Hopkinson pressure bar (HPB) technique overcoming the problem that how to simultaneously measure the stress and particle velocity dual information at one Lagrangian position.

However, it is sometimes cannot simultaneously measure the boundary stress and velocity information due to the particularity of experimental setups and materials. In view of this situation, a more convenient new method of Lagrangian analysis (called as "nv+T0" for short) is proposed based on the measurements of a series of particle velocity waves and the pre-known zero

Corresponding author: dingyuanyuan $@$,nbu.edu.cn 
initial condition in this paper. The outline of this paper is organized as follows. First, a brief introduction of "nv $+\mathrm{T}_{0}$ " Lagrangian analysis method is presented. Secondly, a numerical calculation is carried out to verify the correctness of the proposed Lagrangian method, followed by conclusion in the last Section.

\section{2 "NV+ $\mathrm{T}_{0}$ " Lagrangian analysis method}

In the case of one-dimensional wave propagation, when ignoring the influences of heat conduction, body force and internal power source, mass and momentum conservation equations in Lagrangian coordinates are given by

$$
\frac{\partial v}{\partial X}=\frac{\partial \varepsilon}{\partial t}
$$

and

$$
\rho_{0} \frac{\partial v}{\partial t}=\frac{\partial \sigma}{\partial X}
$$

where $\sigma, \varepsilon, v$ are stress, strain and particle velocity, respectively; $X$ and $t$ are Lagrangian coordinate and time, respectively; $\rho_{0}$ is the initial density of specimen. Here, the stress and strain are positive for tensile case, and negative for compressive case.

It is obvious that the mass conservation equation establishes a relation between strain $\varepsilon$ and particle velocity $v$, while the momentum conservation equation provides a relation between stress $\sigma$ and particle velocity $v$. Thus, the relationship of strain and stress can be built with the aid of velocity field. Nevertheless, those quantities are connected by their first order derivatives, which means initial or boundary conditions should be provided to solve this problem.

Considering the case that particle velocity wave profiles $v\left(X_{i}, t\right)$ are measured by $n$ velocity gauges at $n$ Lagrangian coordinates $X_{i}$, as shown in Fig. 1. To describe the characteristics of wave propagation more accurately, the path-line method, first proposed by Grady, is used to aid the derivative computation of Lagrangian analysis for the large error in the partial derivatives containing $X$. The construction of the pathline is as follows. First, divide the velocity field into several zones by the feature points (such as the inflection point, maximum/minimum point) in these velocity time history curves. Secondly, insert nodes with same number in each velocity curve in the same zone, thus each velocity curve has $N$ nodes. By this means, the velocity of each node can be expressed as $v_{i j}$, where $i$ represents the number of measured Lagrangian position $X_{i}$, and $j$ is the number of node in each velocity profile. Finally, the path-line can be constructed by connecting the corresponding nodes in each velocity profile by a smooth curve. $N$ path-lines can be obtained and link together the entire velocity field.

The total differential form of $\varphi(X, t)$ along the Pathline $p$ is

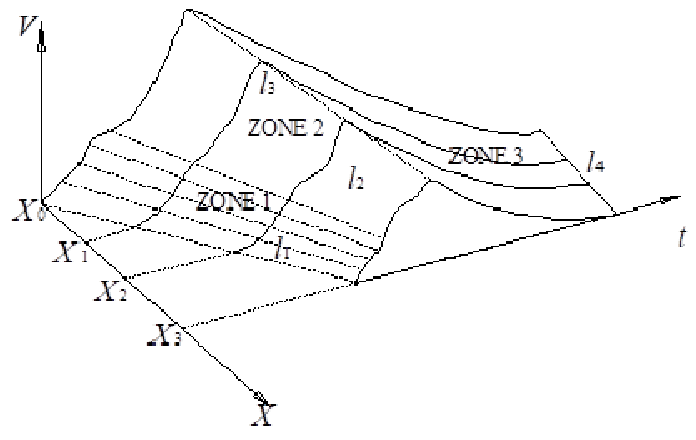

Fig. 1. Schematics of path-line method.

$$
\left.\frac{d \varphi}{d X}\right|_{p}=\left.\frac{\partial \varphi}{\partial X}\right|_{t}+\left.\left.\frac{\partial \varphi}{\partial t}\right|_{X} \frac{d t}{d X}\right|_{p}
$$

where $\varphi$ can represent stress, strain, particle velocity and other physical quantities.

$$
\left.\frac{d \sigma}{d X}\right|_{p}=\left.\frac{\partial \sigma}{\partial X}\right|_{t}+\left.\left.\frac{\partial \sigma}{\partial t}\right|_{X} \frac{d t}{d X}\right|_{p}
$$

Combining the momentum conservation equation (Eq. (2)), we have

$$
\rho_{0} \frac{\partial v}{\partial t}=\frac{\partial \sigma}{\partial X}=\left.\frac{d \sigma}{d X}\right|_{p}-\left.\left.\frac{\partial \sigma}{\partial t}\right|_{X} \frac{1}{X^{\prime}}\right|_{p} .
$$

For the first path-line $p_{1}$ which connects the points $\left(X_{i}, 0\right)$ at initial time $t=0$ for each Lagrangian coordinates $X_{i}(i$ $=1,2, \ldots n)$, the known initial condition provides:

$$
\left.\sigma\right|_{t=0}=\left.v\right|_{t=0}=\left.\varepsilon\right|_{t=0}=0 \text { and }\left.\frac{d \sigma}{d X}\right|_{p_{0}}=0 .
$$

Thus, for the first path-line $p_{1}$, Eq. (5) reduces as

$$
\left.\frac{\partial \sigma}{\partial t}\right|_{X}=-\left.\rho_{0} X^{\prime}\right|_{p_{0}} \frac{\partial v}{\partial t} .
$$

The corresponding difference equation can be written as

$$
\sigma_{i, 2}=\sigma_{i, 1}-\rho_{0}\left(\frac{d X_{i, 1}}{d t} \frac{\partial v_{i, 1}}{\partial t}\right)\left(t_{2}-t_{1}\right)
$$

By numerical integral operations, the stress $\sigma$ on the second path-line $p_{2}$ can be determined by Eq. (8). Therefore, the stresses on the following Path-lines can be obtained by Eq. (5), and its difference equation is

$$
\sigma_{i, j+1}=\sigma_{i, j}+\left.\left(\left.\frac{d \sigma_{i, j}}{d X}\right|_{p}-\rho_{0} \frac{\partial v_{i, j}}{\partial t}\right) \frac{d X_{i, j}}{d t}\right|_{p}\left(t_{j+1}-t_{j}\right) .
$$

Thus, the total stress field $\sigma\left(X_{i}, t\right)$ can be finally determined.

Similarly, by utilizing the total derivative of $\varepsilon$ along the path-line, Eq. (1) can be re-written as 


$$
\left.\frac{\partial \varepsilon}{\partial t}\right|_{X}=\left.\frac{d v}{d X}\right|_{p}-\left.\frac{\partial v}{\partial t} \frac{1}{X^{\prime}}\right|_{p}
$$

and its difference equation is

$$
\varepsilon_{i, j+1}-\varepsilon_{i, j}=\left(\frac{d v_{i, j}}{d X}-\frac{\partial v_{i, j}}{\partial t} \frac{d t_{i, j}}{d X}\right)\left(t_{j+i}-t_{j}\right) .
$$

Thus, the stress field and strain field can be obtained by Eqs. (8), (9) and (11) just need to know a few particle velocity profiles, and such a new method of Lagrangian analysis is called " $\mathrm{nv}+\mathrm{T}_{0}$ " for short.

\section{Numerical simulation}

The feasibility of this new ' $1 \mathrm{sv}+\mathrm{T}_{0}$ ' Lagrangian analysis method is evaluated by a numerical simulation. Polymethyl methacrylate (PMMA), a typical ratedependent viscoelastic material, is taken as the example, and its constitutive relation can be well described by Zhu-Wang-Tang (ZWT) nonlinear viscoelastic constitutive equation. The ZWT equation is reduced to simplified equation when the strain is less than $1 \%$, which is

$$
\sigma=E_{a} \varepsilon+E_{2} \int_{0}^{t} \varepsilon(\mathrm{t}) \exp \left(-\frac{t-\tau}{\theta_{2}}\right) \mathrm{d} \tau
$$

where $E_{\mathrm{a}}$ is the elastic constant for elastic equilibrium response, $E_{2}$ and $\theta_{2}$ are the elastic constant and relaxation time for the high-frequency viscoelastic Maxwell element. The material parameters of PMMA considered here are from the literature [9], where density $\rho_{0}=1190 \mathrm{~kg} / \mathrm{m}^{3}, E_{\mathrm{a}}=2.94 \mathrm{GPa}, E_{2}=3.07 \mathrm{GPa}$ and $\theta_{2}=$ $95.4 \mu \mathrm{s}$. The boundary condition in the numerical simulation is constant strain loading, and its particle velocities at four Lagrangian positions are shown in Fig.2, which are set as known condition for the analysis of " $\mathrm{nv}+\mathrm{T}_{0}$ " Lagrangian method.

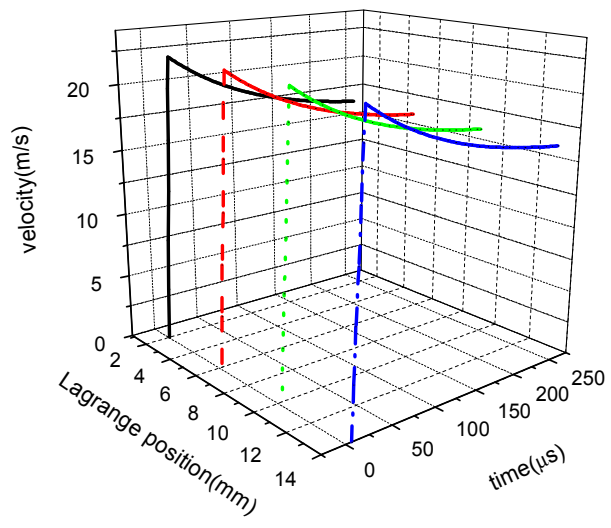

Fig. 2. Velocity-time curves at different Lagrange positions.
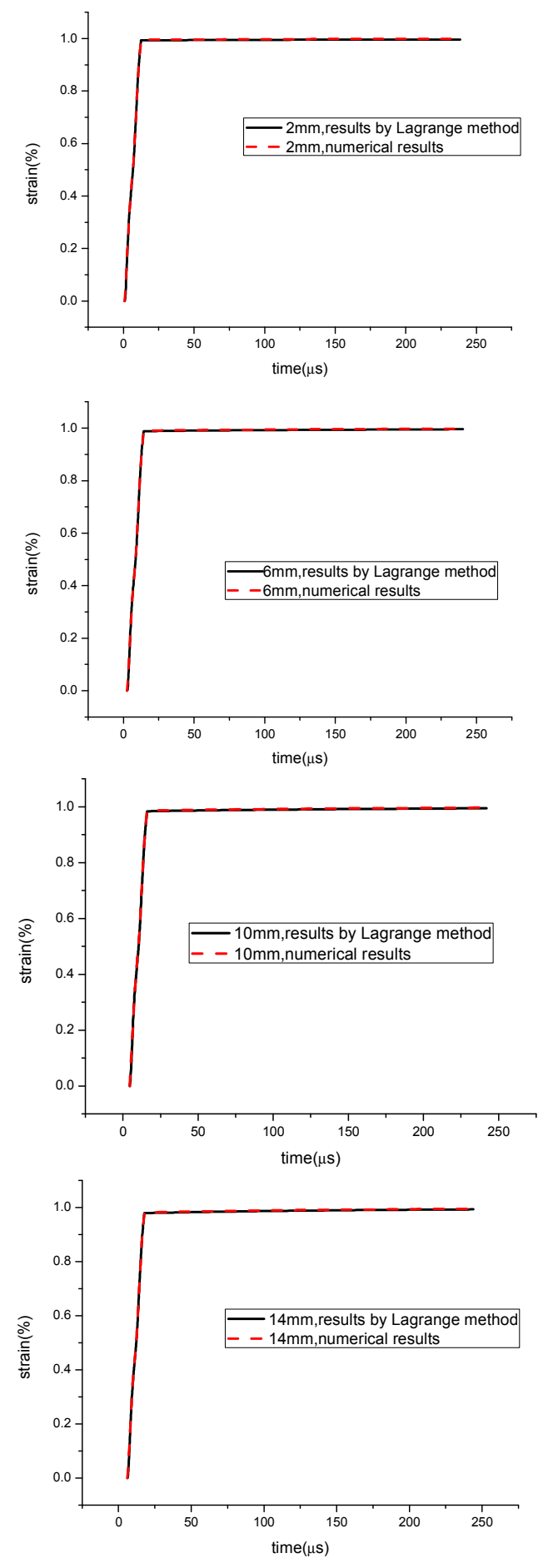

Fig. 3. Strains by numerical calculation (dotted lines) and "nv $+\mathrm{T}_{0}$ " Lagrange analysis (solid lines) at different Lagrange positions.

Thus, the strain and stress results of PMMA can be directly obtained by the "nv $+\mathrm{T}_{0}$ " Lagrange analysis method (8), (9), and (11)), as shown in Figs 3 and 4. The comparison results revealed that the numerical results are in good agreement with the results determined by the "nv $+\mathrm{T}_{0}$ " Lagrange analysis method. A tiny difference of stress results at the end time can be found and this may be caused by the differential accuracy. 

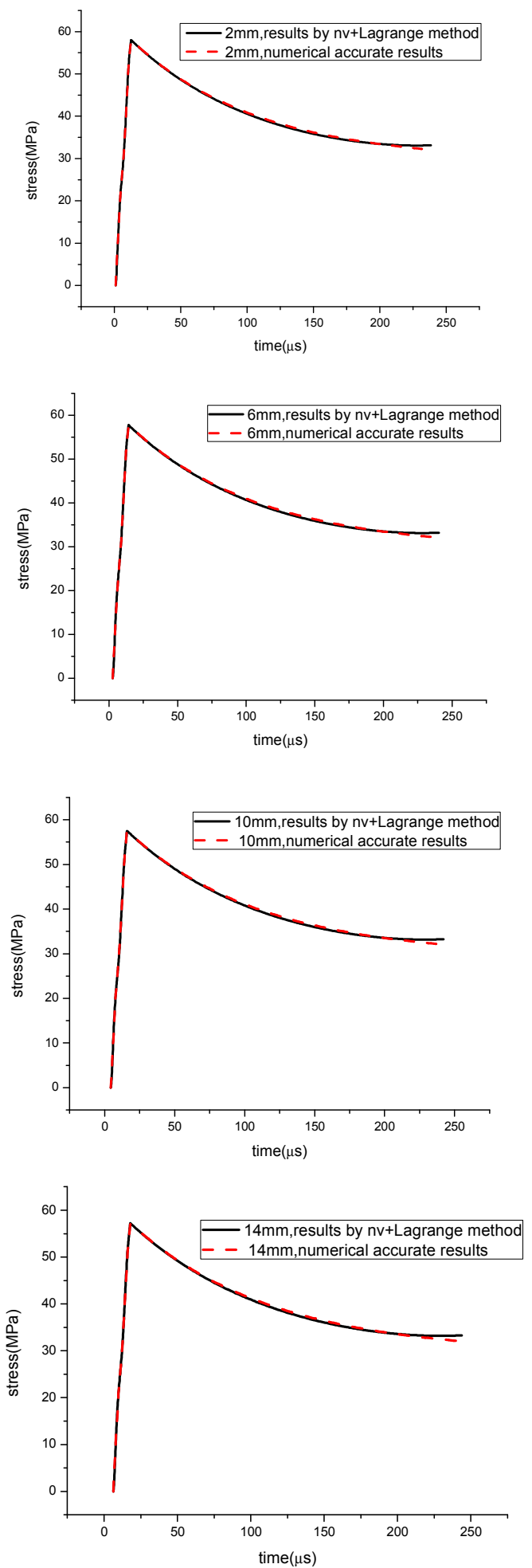

Fig. 4. Stresses by numerical calculation (dotted lines) and "nv $+\mathrm{T}_{0}$ " Lagrange analysis (solid lines) at different Lagrange Lagrange positions.

\section{Conclusions}

The difficulty of the traditional Lagrangian analysis can be attributed to how to simultaneously measure the particle velocity and stress curves at the same Lagrange position, but sometimes it is often not possible to measure these two quantities simultaneously. In order to solve this problem, this paper presents the " $\mathrm{nv}+\mathrm{T}_{0}$ " Lagrange analysis without any pre-assumption for the constitutive model and any requirement of combining gauge. Just based on a set of particle velocity curves under Lagrangian coordinates, the stress-strain relationship of the material can be directly obtained according to the path-line method combined with zero initial conditions, without the need for the boundary stress. A typical rate-dependent viscoelastic material (PMMA) is taken as the numerical simulation to verify the correctness of the "nv $+\mathrm{T}_{0}$ " Lagrange analysis method, and the results are in good agreement.

This work is supported by the National Natural Science Foundation of China (Projects Nos. 11702152), and the Zhejiang provincial key science and technology innovation team (Project No. 2013TD21) program.

\section{References}

1. L.L. Wang, Foundations of Stress Waves (Elsevier, Amsterdam, 2007)

2. H. Kolsky, Proc. Phys. Soc. B62, 676 (1949)

3. L.M. Yang, V.P.W. Shim, Int. J. Impact Eng. 31(2), 129 (2005)

4. R. Fowles, J. Appl. Phys. 41, 2740-2741 (1970)

5. M. Cowperthwaite, R. F.Williams, J. Appl. Phys. 42, 456 (1971)

6. D.E. Grady, J. Geo. Res. 8, 1299 (1978)

7. X.L. Li, Explosion Shock Waves 5, 45 (1985)

8. H. Chai, Z.P. Tang, Explosion Shock Waves 14, 332 (1994)

9. L.L. Wang, J. Zhu, H.W. Lai, Strain 47, 173 (2011) 